possessed sufficient explosive energy. No one supposes that the volcanoes on the earth at present eject the fragments which will constitute future meteorites, but it seems probable that the earth may be now slowly gathering back in these quiet times the fragments she ejected in an early stage of her history.

Assuming, therefore, that the meteorites have had a quasivolcanic origin on scme considerable celestial body, I am led to agree with those who believe that most probably that body is the earth.

ROBERT S. BALL

\section{RECENT RESEARCHES ON ABSORPTION SPECTRA}

THE numerous absorption spectra of soluble substances which have been described hitherto, have referred as a rule to the solutions of the substances, and but rarely to the solid substances themselves. It is true that certain differences were remarked between the spectra of certain solutions, those of uranium and didymium salts, for instance, and the spectra of the solid salts; yet, on the whole, these differences were so slight that it was generally believed that the spectra were essentially the same. On the other hand experiments had shown that the spectra of solutions differed according to the dissolving medium; indeed Herr Kundt established the fact that the absorption band of a substance in solution lies the nearer to the red end of the spectrum the stronger the dispersion of the dissolving medium. In these experiments the fact seems to have been overlooked that when changing the dissolving medium often the whole character of the spectrum is changed, so that comparison with the former one becomes extremely difficult. Close investigation of these differences was therefore an important desideratum, both for the theory of absorption spectra as well as for practical absorption spectrum analysis.

In the Monthly Report of the Berlin Academy of Sciences, Herr Vogel has recently published the results of such investigations, to which he was led by the remarkable differences between the spectra of solid and those of dissolved substances which he had observed in the case of certain pigments.

For the examination of these absorption spectra Herr Vogel used instruments of but moderate dispersion, which allow of an casier survey of the whole spectrum, and consequent judgment of its general character, than is the case with strongly dispersing spectroscopes. The absorption spectra of solid salts and pigments were obtained from thin layers of these substances, prepared upon glass plates, through evaporation of a few drops of solution. Herr Vogel reproduces the spectra he observed on two plates, which at once show not only thedifferences in the spectra of one and the same solid substance and its solution, but frequently an extraordinary coincidence in the position of the absorption bands belonging to totally different substances (for instance in nitrate of uranium and permanganate of potash). Of several substances, such as iodine, hyponitric acid, and indigo, the spectrum of the vapour is also given for comparison, and in most cases the aqueous, alcoholic, and some other solution of each substance has been examined.

Without entering into the highly interesting details for which we must refer our readers to the original paper, we confine ourselves to stating the results of Herr Vogel's researches, which are the following: I. Considerable differences exist between the spectra which a substance gives in the solid, liquid, or dissolved and gaseous state. Characteristic bands which are shown in the spectrum of one state are either not reproduced in that of the other (this is the case with chrome alum, chloride of cobalt, iodine, bromine, naphtaline red, fuchsine, indigo, cyanine, aniline blue, methyl violet, eosine, carmine, purpurine, alizarine, santaline), or they reanpear in a different position, or different intensity (examples - Yitrate of uranium, permanganate of potash, hyponitric acid, alcanna red). Sulphate of copper and chlorophyll show the same absorption both in the dissolved and in the solid state.

2. The spectra given by the same substance when dissolved in different media are the same in some cases (purpurine in alcohol or sulphide of carbon, aldehyde green in water or alcohol, methyl violet and indigo-sulphuric acid in water or amylic alcohol); in other cases they differ only in the position of bands (chloride of cobalt, fuchsine, coralline, eosine and iodine green (chloric character is totally different, so that no point of coincidence remains (iodine in sulphide of carbon or alcohol, naphtaline, aniline blue, purpurine, hæmatoxyline, brasiline in water or alcohol).

3. The rule established by Kundt, viz., that the absorption bands of a body in solution lie the nearer towards the red end of the spectrum the greater the dispersion of the dissolving medium is in the region of the bands, is not confirmed in many cases; on the contrary, in some instances the absorption bands move towards the blue in a solution of greater dispersion (nitrate of uranium and blue chloride of cobalt in water and alcohol); in other cases their position remains unaltered for various. media (hyponitric acid in air and benzol, indigo-sulphuric acid and methyl violet in water and amylic alcohol, aldehyde green in water and alcohol, purpurine in sulphide of carbon and alcohol). In some cases a great difference in the sense of Kundt's rule becomes apparent, while in others for the same spectral region but a very trifling one appears, according to the nature of the pigment (coralline and fuchsine). Indeed it happens sometimes that certain bands are in the same position with different dissolving media, while others which are simultaneously visible are displaced (nitrate of uranium in water and alcohol, oxide of cobalt in glass and in water, protonitrate of uranium in neutral solution and in a solution of oxalic acid, chlorophyll in alcohol and ether).

4. The position of absorption bands in the spectra of solid and dissolved bodies may be only exceptionally deemed characteristic for any certain body. Totally different bodies show absorption bands in exactly the same position (solid nitrate of uranium and permanganate of potash in the blue; naphtaline red and coralline in the yellow; indigo, aniline blue, and cyanine in the orange aldehyde green and malachite green in the orange). Closely related substances sometimes show remarkable differences in the position of their bands under prefectly equal conditions (solid uranium salts).

5. The rule set up for absorption spectra, "each body has its own spectrum," can be admitted only with great restrictions. The great number of polychromatic substances show different colours and different spectra in the solid state, according to the direction in which they are observed. Most other bodies show different spectra in the solid state from those of their solutions, and in the latter case again different ones according to the dissolving medium, and the question arises which of all these spectra is the body's "own" spectrum.

The most important difference of the spectra of elements in a state of incandescent vapour, the position of the spectral lines, ceases to be characteristic in the case of absorption spectra of liquid and solid bodies. In the latter spectra, however, the characteristic differences shown by the spectra of incandescent vapours cannot be expected. It is known that metals, which give such remarkably different spectra in the state of incandescent vapours, all give qualitatively the same spectrum as incandescent liquids or solids, viz., a continuous one; for this reason the absorption spectra of these bodies cannot show any remarkable characteristic differences, whatever quantitative differences may become apparent with regard to the absorbed colours. If these well-known facts show that already with regard to elements the laws applying to the spectra of gases do not apply to those of liquids and solids, then : Herr Vogel's investigations prove that in the case of compound bodies simple relations between the spectra of their different aggregate states are still less frequent and occur only exceptionally.

The analysis of absorption spectra therefore is based not so much upon the recognition of the position of the absorption bands of a substance, as upon the changes in the spectra of the same body which take place under the influence of various dissolving media and reagents. Thus cyanine and aniline blue dissolved in alcohol give a very similar spectrum, dissolved in water a totally different one. The absorption bands of oxyhrmoglobine disappear with reducing agents; those of carmine, which are in a similar position, do not; the band of brasiline disappears when acetic acid is added to the solution, that of fuch sine does not, \&c., \&c.

The position of bands becomes more characteristic for the recornition of a body, if the latter shows several absorption bands. But even here we should go too far if from the accidental coincidence in the position of bands of two different bodies we were to draw conclusions regarding any similarity or chemical identity between them (this has indeed been done in certain cases, particularly with blood and chlorophyll). A conclusion regarding such similarity or identity is only justified if the same bands show equal intensities and analogous changes under the influence of the same reagents. 